\title{
Four-well tunneling states and elastic response of clathrates
}

\author{
I. Zerec,${ }^{1}$ V. Keppens, ${ }^{2}$ M. A. McGuire ${ }^{3}$ D. Mandrus, ${ }^{4}$ B. C. Sales,${ }^{4}$ and P. Thalmeier ${ }^{1}$ \\ ${ }^{1}$ Max-Planck-Institute for Chemical Physics of Solids, D-01187 Dresden, Germany \\ ${ }^{2}$ Dept. of Materials Science and Engineering, The University of Tennessee, Knoxville, TN 37996 \\ ${ }^{3}$ Dept. of Physics and Astronomy, The University of Mississippi, University, MS 3867t \\ ${ }^{4}$ Condensed Matter Sciences Division, Oak Ridge National Laboratory, P.O. Box 2008, Oak Ridge, TN 37831
}

(Dated: November 17, 2018)

\begin{abstract}
We present resonant ultrasound elastic constant measurements of the clathrate compounds $\mathrm{Eu}_{8} \mathrm{Ga}_{16} \mathrm{Ge}_{30}$ and $\mathrm{Sr}_{8} \mathrm{Ga}_{16} \mathrm{Ge}_{30}$. The elastic response of the Eu clathrate provides clear evidence for the existence of a new type of four-well tunneling states, described by two nearly degenerate four level systems (FLS). The FLS's are closely linked with the fourfold split positions of Eu known from neutron diffraction density profiles. Using a realistic potential we estimate the tunneling frequencies and show that the energy gap between the two FLS's is of the same order as the Einstein oscillator frequency. This explains why the observed harmonic oscillator type specific heat is not modified by tunneling states. In addition the quadrupolar interaction of FLS's with elastic strains explains the pronounced depression observed in elastic constant measurements. In the case of the Sr clathrate, we show that the shallow dip in the elastic constant $c_{44}$ is explained using the same type of quadrupolar interaction with a soft Einstein mode instead of a FLS.
\end{abstract}

PACS numbers: $62.30 .+\mathrm{d}, 65.40 .-\mathrm{b}, 66.35 .+\mathrm{a}$

Clathrate compounds are composed of polyhedral cages with 12-16 faces formed by Si, Ge or Ga atoms [1]. The oversized cages accommodate guest atoms like Ba, $\mathrm{Sr}$, or Eu which exhibit large amplitude anharmonic motion. This leads to a strong scattering of acoustic phonons and a small thermal conductivity. In many cases the electrical conductivity remains high and the resulting large thermoelectric figure of merit has led to sustained interest in this class of compounds. The glass-like low temperature thermal conductivity [2, 3] and ultrasound attenuation [4, 5] have been interpreted as evidence for the existence of tunneling states. These are commonly discussed within a generic two well potential model leading to two level system (TLS). The thermal conductivity is more glass-like for clathrates with larger atomic displacement parameters (ADP) of the guest atoms. For the clathrates discussed here the ADP's are very large. The nuclear density map for $\mathrm{Eu}(2)$ in $\mathrm{Eu}_{8} \mathrm{Ga}_{16} \mathrm{Ge}_{30}$ clearly shows the distinct symmetrically related four maxima, away from the cage center [6, 7], leading to the fourfold split site model. Large static ADP for $\operatorname{Sr}(2)$, and considerably smaller for $\mathrm{Ba}(2)$, in $\mathrm{Sr}_{8} \mathrm{Ga}_{16} \mathrm{Ge}_{30}$ and $\mathrm{Ba}_{8} \mathrm{Ga}_{16} \mathrm{Ge}_{30}$, respectively, point also to the split site model [3, 6, 8], although the nuclear density maps do not show distinct fourfold maxima.

In this letter we present elastic constant measurements on $\mathrm{X}_{8} \mathrm{Ga}_{16} \mathrm{Ge}_{30}(\mathrm{X}=\mathrm{Eu}, \mathrm{Sr})$ that show anomalies that cannot be naturally explained within a generic TLS model. Instead we propose two new models that we feel are physically appealing because they draw their inspiration from the nuclear density map results.

For $\mathrm{Eu}_{8} \mathrm{Ga}_{16} \mathrm{Ge}_{30}$ we introduce a four-well potential model with the proper symmetry of split sites that describes a rotor type tunneling motion. We show that for appropriate potential parameters two partially degener- ate FLS's exist. This model explains both the specific heat and elastic anomalies and is compatible with neutron diffraction.

For $\mathrm{Sr}_{8} \mathrm{Ga}_{16} \mathrm{Ge}_{30}$ the elastic response is explained using the same quadrupolar interaction with elastic strains, but with local $\mathrm{Sr}$ vibrations. This model may be also considered in other compounds with soft local modes, such as skutterudites, where a similar shallow dip in the elastic constant is observed [9], without pronounced feature in the specific heat.

The elastic constants reported in this work were measured using Resonant Ultrasound Spectroscopy (RUS) [10]. RUS is a novel technique for determining the elastic moduli of solids, based on the measurement of the resonances of a freely vibrating body: in a RUS experiment, the mechanical resonances of a freely vibrating solid of known shape are measured, and an iteration procedure is used to 'match' the measured lines with the calculated spectrum. This allows determination of all elastic constants of the solid from a single frequency scan, eliminating the need for separate measurements to probe different moduli. RUS measurements were taken as a function of temperature using a homemade probe that fits in a Physical Property Measurement System (PPMS) from Quantum Design. Heat capacity measurements were also performed in the PPMS using the apparatus from Quantum Design.

The four well potential is modeled, in accord with nuclear density data, as

$$
V(\rho, \phi)=\frac{V_{0}}{\rho^{2}}(1+\cos (4 \phi))+\frac{V_{1}}{\rho^{2}}+\frac{1}{2} K \rho^{2} .
$$

The harmonic oscillator (HO) potential of the last term is modified by the four-well potential term. $\mathrm{V}_{0}$ is the 


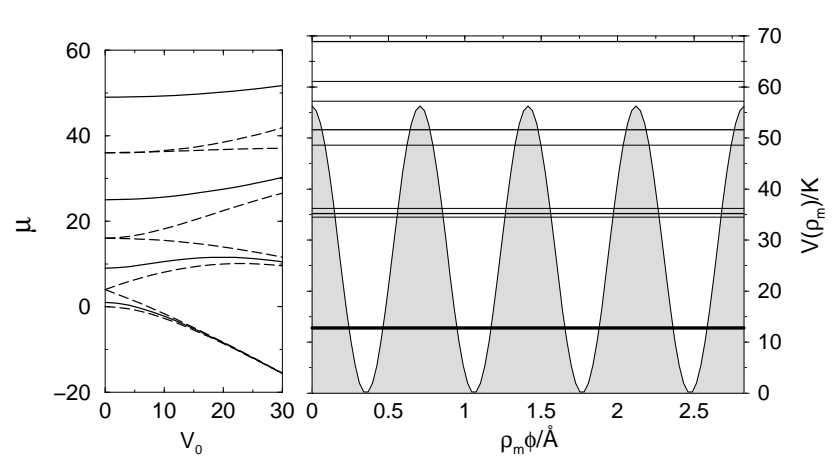

FIG. 1: Left: evolution of $\mu$ with potential barrier $\bar{V}_{0}$. Dashed lines represent nondegenerate (for $\bar{V}_{0}>0$ ) and the solid lines doubly degenerate levels. The formation of lower $\mathrm{FLS}_{1}$ and upper $\mathrm{FLS}_{2}$, may be followed. Right: eigenvalues $\lambda_{n m}$ and potential $\mathrm{V}(\phi, \rho)$ on the circle $\rho=\rho_{0}$ with parameters appropriate for $\mathrm{Eu}_{8} \mathrm{Ga}_{16} \mathrm{Ge}_{30}$. Lower $\mathrm{FLS}_{1}$ : fat line, doublets: thick line, nondegenerate state: thin line. Energies in units of $\mathrm{K}$. For every eigenvalue $\mu_{m}$, there is a sequence of equidistant eigenvalues $\lambda_{n, m}$ of the radial equation (Eq 3 separated by $4 \times \Theta / 2=60 \mathrm{~K}$.

potential barrier in the azimuthal direction. The $\mathrm{V}_{1}$ term determines the positions of the minima, located in the directions $\phi=(2 n+1) \pi / 4$, for $n=\{0,1,2,3\}$, at $\rho_{\text {min }}=\sqrt[4]{2 V_{1} / K}$. It also represents the infinite potential barrier across the center of the cage. This means that there is no tunneling through (and no nuclear density in) the center, consistent with the nuclear density map for $\mathrm{Eu}_{8} \mathrm{Ga}_{16} \mathrm{Ge}_{30}$ 6, 7]. The $\mathrm{HO}$ force constant is given by Einstein temperature, $\Theta$, of the soft local mode of the guest atom of mass $\mathrm{M}, K=M\left(k_{B} \Theta / \hbar\right)^{2}$. $\Theta$ may be determined from ADP, Raman, or specific heat data. The wave function may be written, after separation of the variables, as the product of angular and radial parts, $\psi(\rho, \phi)_{n, m}=R(\rho)_{n, m} \Phi(\phi)_{m}$. The former obeys

$$
\frac{\partial^{2}}{\partial \phi^{2}} \Phi_{m}+\left(\mu_{m}-\bar{V}_{0} \cos (4 \phi)\right) \Phi_{m}=0
$$

where $\bar{V}_{0}=\frac{2 M}{\hbar^{2}} V_{0}$ is dimensionless. For $\bar{V}_{0}=0$ eigenvalues $\mu_{m}^{(0)}=m^{2}$, eigenfunctions $\Phi_{m}^{ \pm}(\phi)=\cos m \phi, \sin m \phi$ and angular momentum $m \hbar$ correspond to the free rotor. This has a formal analogy to directional rotors formed by guest molecules in clathrates [12. Introduction of $\bar{V}_{0}$ breaks the full azimuthal symmetry to fourfold symmetry, lifts the twofold degeneracy of states with even values of $m$ and leads to the formation of FLS with increasing $\bar{V}_{0}$, shown in Fig. 1. The radial wave function satisfies

$\frac{1}{x} \frac{\partial}{\partial x} x \frac{\partial R_{n, m}}{\partial x}+\left(\lambda_{n, m}-\frac{\mu_{m}+\bar{V}_{0}+\bar{V}_{1}}{x^{2}}-x^{2}\right) R_{n, m}=0$.

Here, $x^{2}=\left(M K / \hbar^{2}\right)^{1 / 2} \rho^{2}$ is the dimensionless radial

\begin{tabular}{|c|c|c|c|c|c|c|}
\hline Atom & $A$ & $\rho_{0} / \AA$ & $\Theta / \mathrm{K}$ & $\Delta_{1} / \mathrm{K}$ & $\Delta_{2} / \mathrm{K}$ & $\Delta_{3} / \mathrm{K}$ \\
\hline $\mathrm{Sr}[11]$ & 87.6 & 0.35 & 50 & 0.11 & 2.23 & 56 \\
$\mathrm{Ba}[3]$ & 137.3 & 0.20 & 70 & 2.19 & 4.45 & 110 \\
$\mathrm{Eu}[3,6,11]$ & 152.0 & 0.43 & 30 & 0.05 & 0.85 & 22 \\
\hline
\end{tabular}

TABLE I: The parameters of "guest" atoms for the three clathrates. $A$ is the atomic mass number. $\Delta_{1}, \Delta_{2}$, and $\Delta_{3}$ are the tunneling energies of the $\mathrm{FLS}_{1}, \mathrm{FLS}_{2}$, and the gap between the two, respectively, obtained for $\bar{V}_{0}=30$. $\rho_{0}$ for $\mathrm{Eu}$ is the average from the three references. (In Ref. [3] the position for $\mathrm{Ba}(2)$ is incorrect. Here the correct $\rho_{0}$ is used.)

coordinate and $\lambda=2 E /\left(k_{B} \Theta\right)$ denotes dimensionless eigenvalues

$$
\lambda_{n, m}=2\left(\sqrt{\mu_{m}+\bar{V}_{0}+\bar{V}_{1}}+1\right)+4 n .
$$

The maxima of the radial wave functions correspond to the nuclear density maxima. The tunneling splitting $\Delta_{1}$ in units of Kelvin for the lowest $\mathrm{FLS}_{1}$ is given by

$$
\Delta_{1} \approx \frac{\hbar^{2}}{2 M k_{B} \rho_{0}^{2}}\left(\mu_{1}\left(\bar{V}_{0}\right)-\mu_{0}\left(\bar{V}_{0}\right)\right) .
$$

Here, $\rho_{0}$ is the split site radial distance from the center, corresponding to the maximum of the $n=0$ radial wave function. The tunneling frequency $\Delta_{2}$ of the upper $\mathrm{FLS}_{2}$ and its energy relative to the lower one, $\Delta_{3}$, may be calculated using (4). $\Delta_{3}$ is a measure for the size of the azimuthal corrugation in the radial minimum. The low lying energy levels for the case of Eu clathrate, are shown in Fig. [1] The parameters for the three clathrates are summarized in Table \. We note that the nuclear density profiles for $\mathrm{Sr}$ and $\mathrm{Ba}$ do not correspond to the FLS wave functions presented in the right part of Fig. 2 Our new model of azimuthal four-well tunneling enforces a re-interpretation of low temperature properties in Eu clathrate.

Using the eigenvalues from (3), the specific heat, $\mathrm{C}(\mathrm{T})$, may be numerically calculated. For the free rotor $\left(\bar{V}_{0}=\right.$ 0 ) closely spaced levels (Fig. (1) lead to a constant $\mathrm{C}(\mathrm{T})$ for moderately low T (Eq. 3 and Fig. 22). Turning on the four well term, $\bar{V}_{0}$, leads to the "bunching" of the levels into two FLS's, with large energy gap $\Delta_{3}$ between them. Since $\Delta_{3} \simeq \Theta$ for Eu potential parameters and $\Delta_{1}$ is very small, $\mathrm{C}(\mathrm{T})$ for the pure HO and FLS model deviate very little. They are equally acceptable, in contrast to the conventional TLS model. In addition, the contribution of guest atoms to the total $\mathrm{C}(\mathrm{T})$ at high temperatures is only around $10 \%$, so that the deviations in the high temperature region are negligible, as seen in Fig. 2

Now we show that our new FLS model in addition leads to characteristic elastic constant depressions observed in $\mathrm{Eu}_{8} \mathrm{Ga}_{16} \mathrm{Ge}_{30}$ by RUS measurements which the pure HO model cannot explain. Assuming a quadrupolar interaction $V \cos (2 \phi)$ of FLS's with the lattice, the matrix 

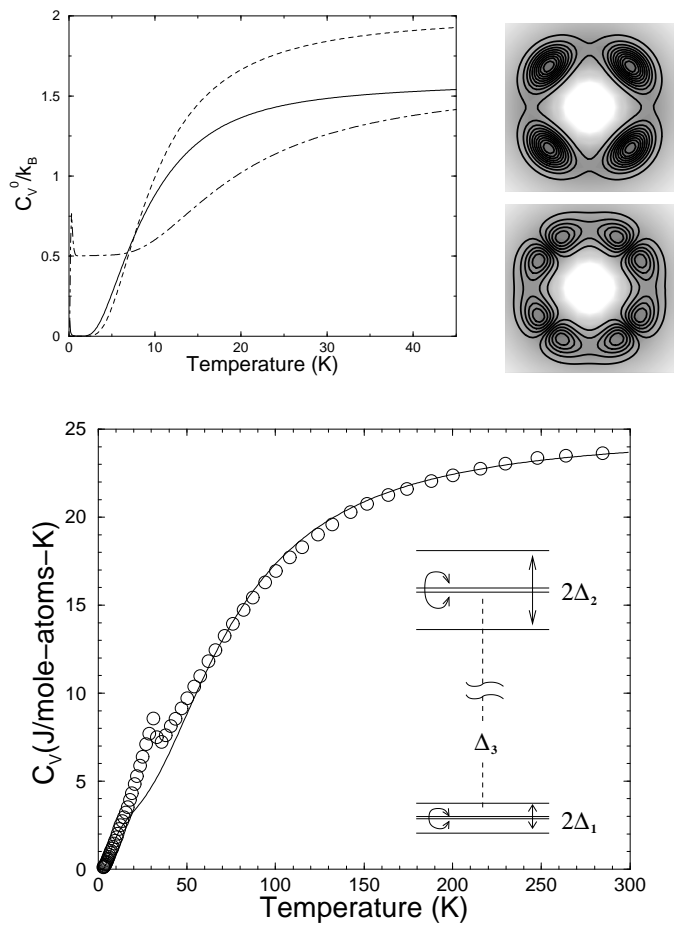

FIG. 2: Top left: specific heat contribution $\mathrm{C}_{V}^{0}$ of a guest atom. Dashed line: unperturbed 2D-HO, dash-dotted line: 2D free rotor with $\bar{V}_{1}=307$ and $\bar{V}_{0}=0$, solid line: FLS's with $\bar{V}_{1}=292$ and $\bar{V}_{0}=30$. These parameters are in accord with the case of $\mathrm{Eu}$ (Table凹). Top right: contour plots of the ground state wavefunctions for $\mathrm{FLS}_{1}$ and $\mathrm{FLS}_{2}$. The shaded background corresponds to the four well potential. Bottom: measured specific heat (circles) of $\mathrm{Eu}_{8} \mathrm{Ga}_{16} \mathrm{Ge}_{30}$ from Ref. 6]. Solid line is the calculated total specific heat $\mathrm{C}_{V}$ including the sum of $6 \mathrm{FLS} /$ cell, HO contributions and the Debye term. It is almost identical to the fit using the pure Einstein oscillator [6] for the guest atom Eu. The peak around $30 \mathrm{~K}$ is due to the ferromagnetic phase transition. Inset: schematic representation of $\mathrm{FLS}_{1,2}$. Arrows indicate transitions within each FLS system due to the quadrupolar interaction.

elements between the eigenstates of Eq. (2) can be numerically calculated. The interaction matrix is nearly of block diagonal form with negligible off-diagonal terms, which is represented as direct sum of the two FLS's:

$$
V_{\text {int }}=\left(\begin{array}{rr}
\gamma_{1} X & 0 \\
0 & \gamma_{2} X
\end{array}\right)
$$

with $X=\sigma^{x} \otimes \sigma^{x}\left(\sigma^{x}=\right.$ Pauli matrix $)$ and $\gamma_{1}, \gamma_{2}$ the quadrupolar coupling strengths for FLS 1 and $\mathrm{FLS}_{2}$, respectively $\left(\gamma_{1} \geq \gamma_{2}\right)$. The possible transitions are shown in the inset of Fig. 2. The interaction term in Eq. (5) is coupled to the elastic strain $e_{\mu}$ leading to the total Hamiltonian

$$
H=\frac{1}{2} V c_{\mu}^{0} e_{\mu}^{2}+e_{\mu} V_{i n t}+H_{F L S} .
$$

Here, $c_{\mu}^{0}$ is the background elastic constant, $V$ is the sam- ple volume and $H_{F L S}$ the noninteracting two-FLS Hamiltonian. The renormalised elastic constant is then given by the static quadrupolar susceptibility of the FLS's according to $\Delta c_{\mu}=\left(N_{c} / V_{0}\right) \chi_{Q}$ where $N_{c}=6$ is the number of $\mathrm{Eu}(2)$ atoms per unit cell of volume $V_{0}$ and

$$
\begin{aligned}
\chi_{Q}= & -\sum_{\lambda_{0, m} \neq \lambda_{0, n}} \frac{\left|\left\langle m\left|V_{i n t}\right| n\right\rangle\right|^{2}}{\lambda_{0, n}-\lambda_{0, m}}\left(p_{m}-p_{n}\right) \\
& -\frac{1}{k_{B} T}\left\{\sum_{\lambda_{0, m}=\lambda_{0, n}}\left|\left\langle m\left|V_{\text {int }}\right| n\right\rangle\right|^{2} p_{m}-\left\langle V_{\text {int }}\right\rangle^{2}\right\}(7)
\end{aligned}
$$

Here $p_{m}$ is the occupation factor for the FLS ${ }_{1,2}$ tunneling state $|m\rangle$. The first (Van Vleck) contribution is due to inelastic transitions within FLS 1 and $\mathrm{FLS}_{2}$. The second (Curie) term is due to elastic transitions within the degenerate doublets of both $\mathrm{FLS}_{1}$ and $\mathrm{FLS}_{2}$.

The FLS's interact with the phonon bath. For a sufficiently strong interaction compared to the tunneling energy the incoherent regime is entered. The crossover temperature is given by $T^{*}=\frac{1}{k_{B}} \sqrt{\frac{2 \hbar}{\pi} \sqrt{\rho v^{5} \hbar} \frac{2 \Delta}{\gamma}}[13] . \Delta_{1}$ is an order of magnitude smaller than $\Delta_{2}$ (Table 【) and correspondingly, $\mathrm{T}^{*}$ for the $\mathrm{FLS}_{1}$ is significantly lower. In the coherent regime $\mathrm{T}<\mathrm{T}^{*}$ the change of $\chi_{Q}$ is due to the relative change of potential minima between the four wells. Because $\Delta_{1}$ is small its coherent tunneling states are destroyed by the coupling to the phonon bath already at $\mathrm{T} \sim 1 \mathrm{~K}$. Then the guest atom may be considered as statistically distributed over the four wells reducing the elastic response to that of four independent wells. This is zero if only the constant shift of a single well is considered. In contrast, for Eu parameters $\mathrm{FLS}_{2}$ is on the boundary of the coherent regime in the temperature range of the depression. Therefore only $\mathrm{FLS}_{2}$ contributes to the quadrupolar response, explicitly:

$\Delta c_{\mu}=-\frac{N_{c}}{V_{0} Z(T)} e^{-\frac{\Delta_{3}}{k_{B} T}}\left[\frac{2 \gamma_{\mu 2}^{2}}{\Delta_{2}} \sinh \left(\frac{\Delta_{2}}{k_{B} T}\right)+\frac{2 \gamma_{\mu 2}^{2}}{k_{B} T}\right](8)$

The depression in $\mathrm{c}_{\mu}$ is caused by the thermal depopulation of the relaxational $\mathrm{FLS}_{2}$, described by the exponential factor in front of Eq. (8). Within a conventional TLS model it may only be described by assuming an unreasonably large tunneling frequency, incompatible with the specific heat data, and unequal equlibrium well depths which in the present context is unphysical because it violets the fourfold symmetry.

The results for the Eu clathrate are shown in Fig. 3 . Our fit determines $\gamma_{\mu 2} \approx 0.073 \mathrm{eV}$, with $\Delta_{3} / k_{B}=22 \mathrm{~K}$. This value is used above to estimate $\mathrm{T}^{*}$ and to justify the neglect of the $\mathrm{FLS}_{1}$ contribution. The depression of $\mathrm{c}_{\mu}$ is a direct signature of the quadrupolar coupling to relaxational processes within the upper $\mathrm{FLS}_{2}$. Contrary to TLS model our FLS tunneling model explains both specific heat and elastic constant anomalies in $\mathrm{Eu}_{8} \mathrm{Ga}_{16} \mathrm{Ge}_{30}$ without contradiction and is consistent 


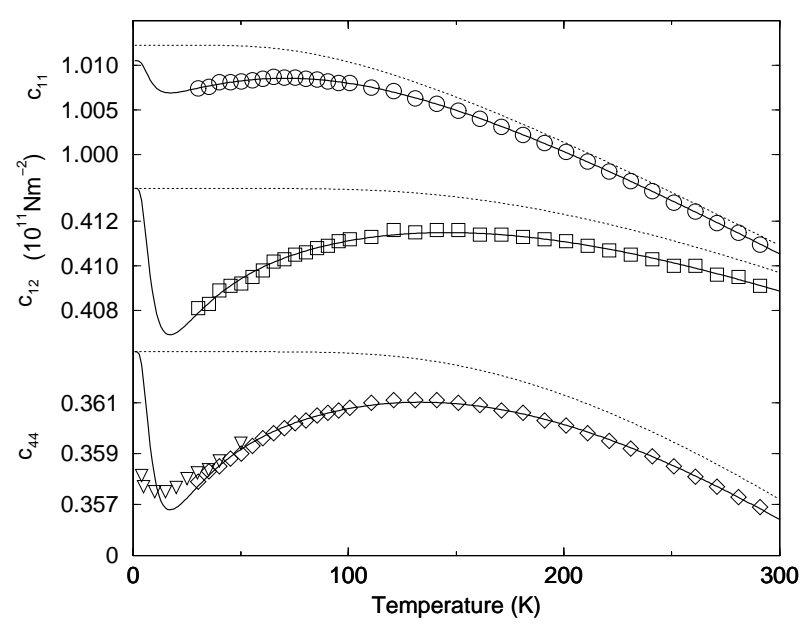

FIG. 3: The fit of the elastic constants of Eu clathrate including interaction with $\mathrm{FLS}_{2}$ (solid thick line). Background variation corresponds to Varshni function [14] (dotted line). The depression with respect to the Varshni background is due to quadrupolar coupling to the upper $\mathrm{FLS}_{2}$. Note the different scales for $c_{\mu}$.

with the nuclear density profile from the neutron diffraction. This presents strong evidence that a new type of FLS tunneling states due to azimuthal tunneling of guest atoms has been discovered in Eu clathrate.

In the case of $\mathrm{Sr}_{8} \mathrm{Ga}_{16} \mathrm{Ge}_{30}$ the split sites are less distinct than in $\mathrm{Eu}_{8} \mathrm{Ga}_{16} \mathrm{Ge}_{30}$, with much of the nuclear density in the center [6], and the four-well potential pre-

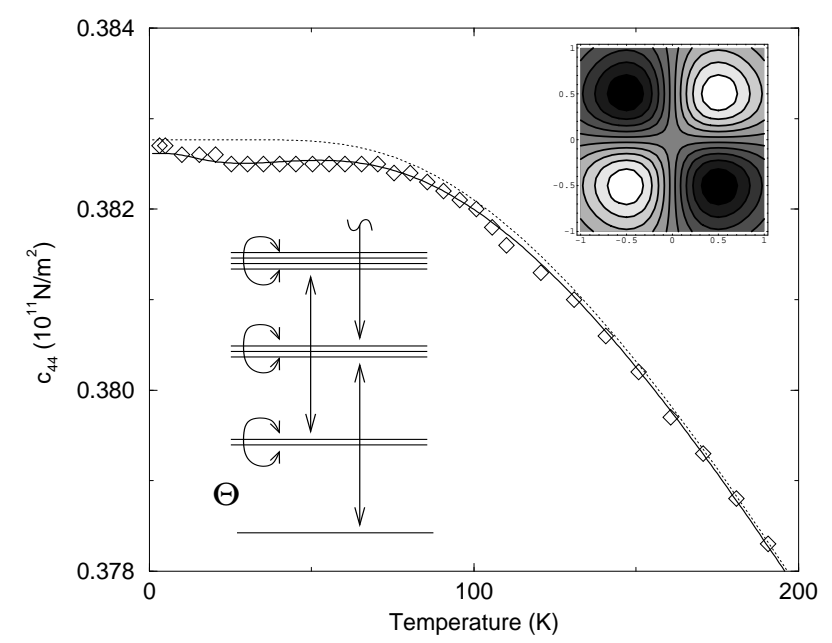

FIG. 4: Elastic constant $\mathrm{c}_{44}$ in $\mathrm{Sr}_{8} \mathrm{Ga}_{16} \mathrm{Ge}_{30}$. Fitted curve (solid thick line) is obtained using the Varshni function for the background (dotted line) and the model of the quadrupole interaction of the local Einstein mode with the elastic strain explained in the text. The interaction constant $\gamma_{H O}=0.015 \mathrm{eV}$. Inset: schematic representation of the transitions between the states of $2 \mathrm{D}-\mathrm{HO}$ due to the quadrupolar interaction. Upper inset: the contour plot of the quadrupolar interaction potential $\mathrm{V}_{\text {int }}^{H O}=\gamma_{H O} \rho^{2} \sin (2 \phi) \exp \left[-\rho^{2} /\left(2 a_{0}^{2}\right)\right], a_{0}=\hbar / \sqrt{K M}$. sented here is not appropriate. Due to the large overlap, as may be deduced from the nuclear density profile, the tunneling frequency may be very large. The split-site model actually has the same agreement with the data as the single site model [8]. Therefore, we propose a different model for $\mathrm{Sr}_{8} \mathrm{Ga}_{16} \mathrm{Ge}_{30}$. We assume that the $\mathrm{Sr}$ atoms sit in the shallow $\mathrm{HO}$ potential that produces a soft Einstein mode. However we now propose a quadrupolar form of interaction which rapidly decays at larger distances from the center. This is a crucial condition for a small dip in the elastic constant to appear due to transitions between the excited degenerate states of 2D-HO. In the upper inset of Fig. 4 this interaction potential is shown. Although the four well guest atom potential for $\mathrm{Sr}$ has not yet developed as in the Eu case, a tendency is present in the model quadrupolar interaction potential which leads to a satisfactory agreement with RUS data in Fig. 固

The RUS measurements were sponsored by NSF grant DMR0206625. Oak Ridge National Laboratory is managed by UT-Battelle, LLC, for the US Department of Energy under contract DE-AC05-00OR22725.

* Present address: Dept. of Physics, Cornell University, Ithaca, NY 14853

[1] G. S. Nolas, G. A. Slack and S.B. Schujman in Semiconductors and Semimetals 69, 255 (2001).

[2] J. L. Cohn, G. S. Nolas, V. Fessatidis, T. H. Metcalf and G. A. Slack, Phys. Rev. Lett. 82, 779 (1999).

[3] S. Paschen, W. Carrillo-Cabrera, A. Bentien, V. H. Tran, M. Baenitz, Yu. Grin and F. Steglich, Phys. Rev. B 64, 214404 (2001).

[4] V. Keppens, B. C. Sales, D. Mandrus, B. C. Chakoumakos, and C. Laermans, Phil. Mag. Lett. 80, 807 (2000).

[5] V. Keppens, M. A. McGuire, A. Teklu, C. Laermans, B. C. Sales, D. Mandrus and B. C. Chakoumakos, Physica B 316-317, 95 (2002).

[6] B. C. Sales, B. C. Chakoumakos, R. Jin, J. R. Thompson and D. Mandrus, Phys. Rev. B 63, 245113 (2001).

[7] B. C. Chakoumakos, B. C. Sales and D. G. Mandrus, J. Alloys Compd. 322, 127 (2001).

[8] B. C. Chakoumakos, B. C. Sales, D. G. Mandrus and G. S. Nolas, J. Alloys Compd. 296, 80 (2000)

[9] V. Keppens, D. Mandrus, B. C. Sales, B. C. Chakoumakos, P. Dai, R. Coldea, M. B. Maple, D. A. Gajewski, E. J. Freeman and S. Bennington, Nature 395, 876 (1998).

[10] A. Migliori, J.L. Sarrao, W.M. Visscher, T.M. Bell, M. Lei, Z. Fisk and R.G. Leisure, Physica B 183, 1 (1993).

[11] G. S. Nolas, T. J. R. Weakley, J. L. Cohn and R. Sharma, Phys. Rev. B 61, 3845 (2000).

[12] A. Würger, Phys. Rev. Lett. 88, 063002 (2002).

[13] A. Würger, From Coherent Tunneling to Relaxation (Springer Tracts in Modern Physics 135, 1996), p. 135.

[14] P. Varshni, Phys. Rev. B 2, 3952 (1970). 